\section{Dermatologische Universitäts-Klinik Jena - Geschichte und Perspektiven}

Zusammenfassung. Die Dermatologische Universitäts-Klinik Jena kann auf eine über 75-jährige Geschichte zurückblicken. 1919 wurde ein Lehrstuhl für Dermatologie und Venerologie eingerichtet, dessen erster Inhaber Bodo Spiethoff war. Unter seiner Leitung wurde 1922-1924 das heutige Kliniksgebäude erbaut. Während jahrzehntelanger Einschränkungen unter der nationalsozialistischen und kommunistischen Diktatur gelang es gleichwohl, die dermatologische Versorgung der Bevölkerung sicherzustellen und immer wieder mit innovativen Leistungen zum Fortschritt der Dermatologie beizutragen. Nach der Wiedervereinigung Deutschlands konnte die räumliche und materielle Ausstattung zeitgemäßen Standards angepasst und unter Nutzung der neuen Möglichkeiten eine konkurrenzfähige dermatologische Forschung und Lehre aufgebaut werden.

Department of Dermatology, University of Jena - History and Perspectives. The Department of Dermatology, University of Jena, was founded in 1919. Bodo Spiethoff became the first Professor and Chairman. Under his leadership the department building still in use today was constructed. During the long years of Nazi and communist dictatorship, Jena dermatologists still succeeded in providing the necessary care for skin patients. They also contributed to the scientific progress of the speciality with innovative research. After the reunification of Germany, the dermatologic department was modernized and equipped with upto-date instrumentation. Research and teaching of dermatology in Jena have reached an internationally competitive level.

\section{Anfänge der Dermatologie in Jena}

Die Jenenser Universität ist eine Gründung der Reformation: Nach Verlust seiner Landesuniversität Wittenberg ließ Friedrich der Weise von Sachsen 1548 aus kaiserlicher Gefangenschaft heraus in Jena eine „Hohe Schule“ gründen, die 1558 zur Universität erhoben wurde. Anlässlich des 175. Geburtstags ihres berühmten akademischen Lehrers (für Geschichte) Friedrich Schiller, wurde die Universität Jena 1934 nach ihm benannt.

Akt Dermatol 2001; 27: $211-214$

(c) Georg Thieme Verlag Stuttgart · New York

ISSN 0340-2541

\section{P. Elsner}

Klinik für Dermatologie und dermatologische Allergologie der Friedrich-Schiller-Universität, Jena (Direktor: Prof. Dr. P. Elsner)
Wie in anderen deutschen Universitäten, kam es auch in Jena im Laufe des 19. Jahrhunderts mit dem beeindruckenden naturwissenschaftlichen Fortschritt zu einer Differenzierung der medizinischen Fachgebiete. Waren Vorlesungen über Hautund Geschlechtskrankheiten vorher Aufgabe von Internisten, aber auch Chirurgen, was als Beleg für die lange Tradition der Dermatochirurgie gelten kann, wurde 1876 von der Weimarischen Regierung eine „außerordentliche Professur für das Gebiet der Inneren Medizin, speziell für Hautkrankheiten, Syphilis und Kinderkrankheiten“ eingerichtet, die mit Hermann Eichhorst (1849-1921) besetzt wurde. Seine Nachfolger waren Paul Fürbringer (1849-1903), Heinrich Unverricht (1853 - 1912) und Oswald Vierordt (1856-1906) [2].

\section{Etablierung eines Dermatologischen Ordinariats und Bau der Klinik}

Bodo Spiethoff, der seit 1905 als Assistent und seit 1908 als leitender Oberarzt in Jena arbeitete, wurde 1911 zum nichtbesoldeten außerordentlichen Professor mit Lehrauftrag für die Dermatologie berufen (Tab.1). 1914 wurde er Direktor der ersten selbständigen Hautklinik, damals mit 110 Betten ausgestattet und in der ehemaligen Lexerschen Chirurgischen Klinik (heute: Klinik für Neurochirurgie) untergebracht. 1919 wurde ein Lehrstuhl für Dermatologie und Venerologie eingerichtet, der 1928 in ein Ordinariat umgewandelt wurde.

Spiethoffs besonderes Verdienst war der Neubau der dermatologischen Klinik an der Erfurter Straße 35. 1922-1924, also mitten in den schwierigen Inflationsjahren, entstand mit großzügiger Unterstützung der Carl-Zeiss-Stiftung eine für damalige Verhältnisse hochmoderne Klinik (Abb.1-3). Modernen Konzeptionen entsprechend war die Anordnung der Bettenstationen in Seitenflügeln um einen zentralen Mitteltrakt mit Funktionsbereichen, Labors und Hörsaal. Letzterer ist nach wie vor für Besucher eindrucksvoll funktional gestaltet: Auf der Nordseite gelegen, ist er von drei Seiten mit Fenstern umgeben, so dass das indirekte Licht für die Beleuchtung der Befunde optimal genutzt werden kann (Abb.4). Die geschmackvolle originale Ausstattung blieb erhalten und wurde vorsichtig renoviert.

Funktionsbereiche waren eine Bestrahlungsabteilung mit Röntgen- und Weichstrahlgeräten und eine zentrale Bäderabteilung. Die Labors waren - wie in Jena nicht anders denkbar mit modernen Zeiss-Mikroskopen ausgestattet. Medizinisch 
Tab. 1 Ordinarien der Dermatologischen Universitäts-Klinik der Friedrich-Schiller-Universität Jena [1]

\begin{tabular}{ll}
\hline Zeitraum & Ordinarius \\
\hline $1911-1934$ & Bodo Spiethoff \\
$1934-1935$ & Walter Schulze \\
$1935-1958$ & Josef Hämel \\
$1959-1965$ & Heinz Langhof \\
$1966-1970$ & Niels Sönnichsen \\
$1970-1993$ & Erwin Günther \\
$1997-$ & Peter Elsner \\
\hline
\end{tabular}

wichtig für die Zeit und heute noch attraktiv für die Patienten war die Nutzung eines großzügigen Parks mit Liegezonen für die Heliotherapie. Wissenschaftlicher Schwerpunkt Spiethoffs war die Venerologie, die er als Landesvorsitzender für Thüringen der Deutschen Gesellschaft zur Bekämpfung der Geschlechtskrankheiten förderte. Daneben interessierten ihn die dermatologischen Symptome bei inneren Erkrankungen, die Blutveränderungen bei Hautkrankheiten und die „unspezifische Reizbehandlung“.

\section{Die Jenaer Dermatologie in den Jahren des National- sozialismus und der DDR}

Eine umfassende historische Aufarbeitung, wie sich die 56 Jahre Diktatur in Mitteldeutschland auf die dermatologische Klinik ausgewirkt und welche Rolle ihre Repräsentanten in den totalitären Systemen gespielt haben, steht noch aus. Die jüngste Debatte um die Person des Jenaer Pädiaters Ibrahim und seine Beteiligung an den Euthanasie-Verbrechen zwingt allerdings zur Vorsicht bei pauschalen Schuld- und Unschuldvermutungen. Die vorliegenden Ausführungen beschränken sich daher auf die sachliche Wiedergabe des historischen Wissensstandes.

Nachdem 1934 Spiethoff einem Ruf nach Leipzig gefolgt war, übernahm Walter Schultze aus Gießen den Lehrstuhl, blieb allerdings nur ein Jahr, um dann nach Gießen zurückzukehren.

1935 folgte Josef Hämel, vom Greifswalder Ordinariat für Dermatologie und Venerologie aus, dem Ruf nach Jena. Schwerpunkte seiner Arbeit waren - wie bei Spiethoff - die sexuell übertragbaren Krankheiten, aber auch Fragen der Pharmakotherapie, Röntgentherapie und der Behandlung der Haut-Tuberkulose, die in den Kriegs- und Nachkriegsjahren dramatisch zunahm. Sein Ordinariat fällt in die Zeit des Nationalsozialismus und, nach Übergabe des zunächst von amerikanischen Truppen besetzten Thüringen an die Sowjets, in die Zeit der kommunistischen Diktatur in Deutschland. 1958 floh Hämel in den Westen Deutschlands und entzog sich so dem Druck der SED-Regierung, als Rektor der Universität Jena an deren Überführung in die „erste sozialistische Universität Deutschlands“ mitzuwirken. Im Folgenden wurde er auf den dermatologischen Lehrstuhl der Universität Heidelberg berufen.

Hämels Nachfolger in Jena war Heinz Langhof, der sich besonders mit der Photodermatologie beschäftigte und ein Porphyrinlabor in Jena aufbaute. Eine bahnbrechende Leistung war

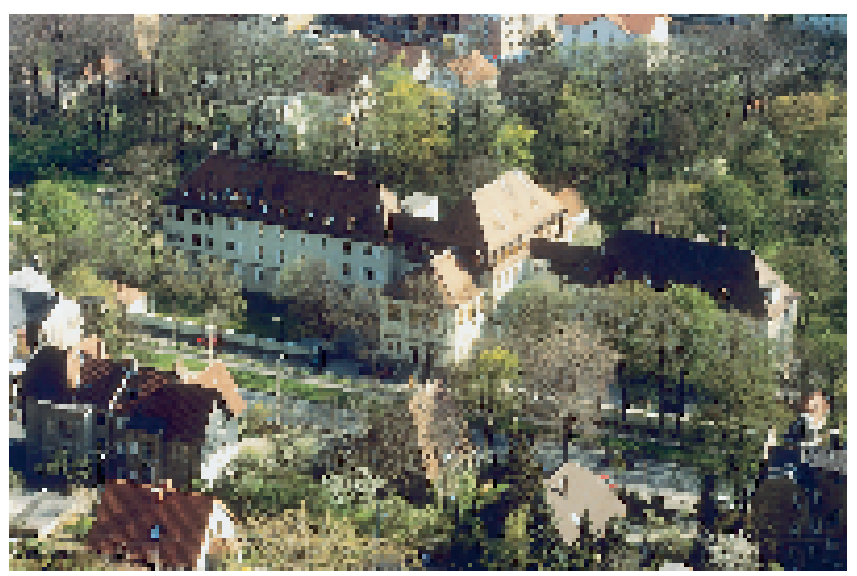

Abb.1 Dermatologische Universitätsklinik Jena: „Luftbild“ von den Jenaer Sonnenbergen.

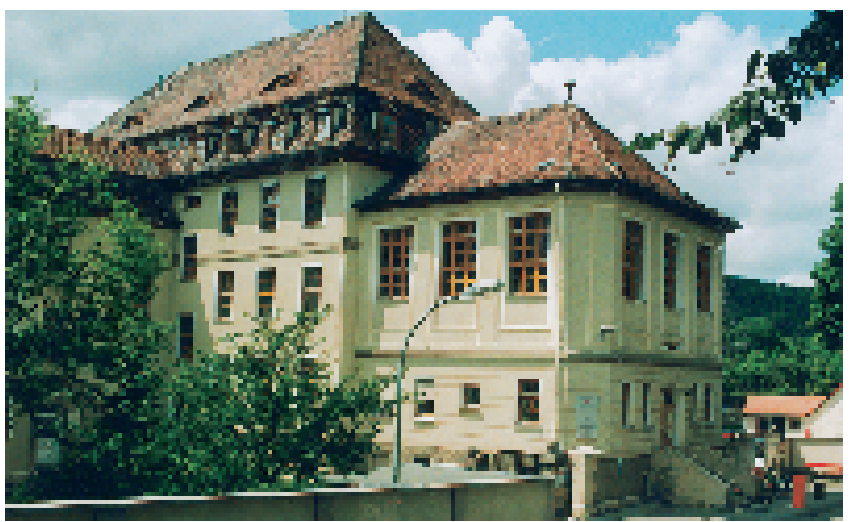

Abb. 2 Dermatologische Universitätsklinik Jena: Mitteltrakt mit Poliklinik (Erdgeschoss) und Hörsaal (Obergeschoss).

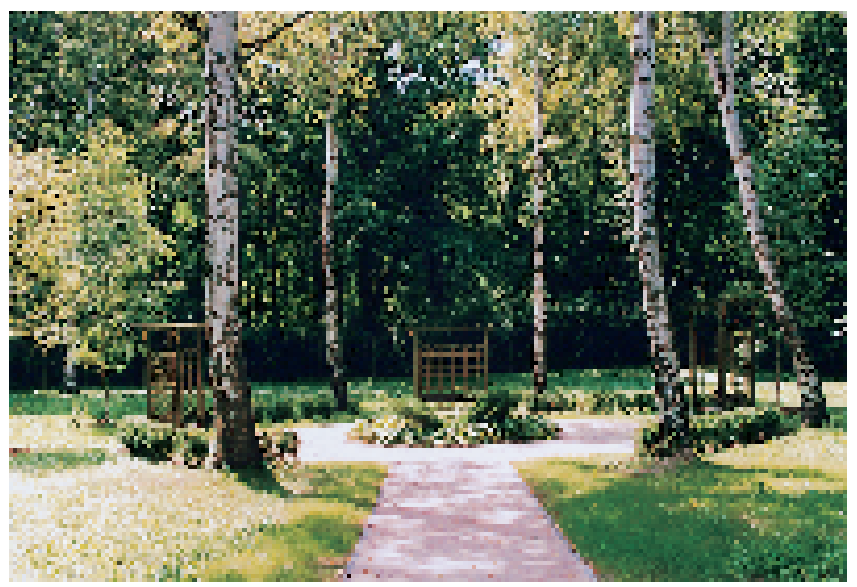

Abb. 3 Dermatologische Universitätsklinik Jena: Neugestalteter Klinikpark.

die Isolierung eines leukotaktischen Faktors aus Psoriasisschuppen, mit der er die Interleukin-Forschung vorwegnahm. Auch als inspirierter akademischer Lehrer war Langhof bei Studenten und Assistenten hochgeschätzt. Umso tragischer war für die Klinik sein früher Freitod 1965. 1966 bis 1970 leitete 


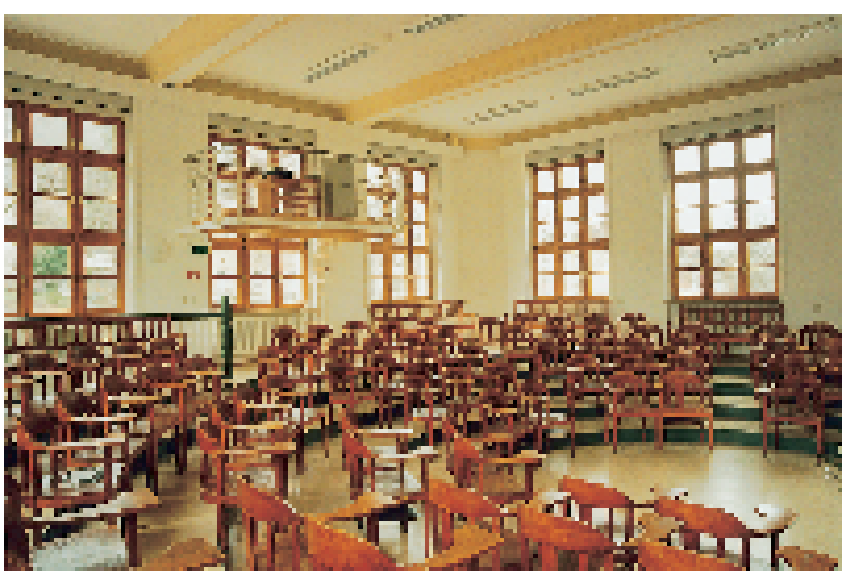

Abb.4 Dermatologische Universitätsklinik Jena: Hörsaal nach der Renovation.

Niels Sönnichsen, aus der Charité kommend, die Jenaer Klinik. Es waren dies höchst produktive wissenschaftliche Jahre, in denen optimale Labormöglichkeiten, so etwa in der Immunfluoreszenzdiagnostik, geschaffen wurden. Es florierten aber auch die Allergologie und Umweltdermatologie, die Andrologie, die Kollagenosenforschung, die Dermatohistologie und die Photodermatologie.

Nach dem Ruf Sönnichsen auf das Ordinariat an der Charité (1970) führte Erwin Günther die Klinik. Sein Schwerpunkt waren die Sexualmedizin, die sexuell übertragbaren Krankheiten und die Andrologie. Unter seiner Leitung habilitierten sich so viele Dermatologen wie nie zuvor: Burhard Knopf (heute Chemnitz), Jochen Meyer (heute Gera), Hans-Jürgen Koch (heute Plauen), Ursula Schmidt, Hans-Henning Scharschmidt und Uwe Wollina.

\section{Die Jenaer Dermatologie nach der Wiedervereinigung Deutschlands}

Nach dem Ausscheiden Erwin Günthers 1993 führte Uwe Wollina die Klinik kommissarisch, bis 1997 Peter Elsner (Zürich) zum Ordinarius berufen wurde. Bereits Anfang der 90er Jahre wurden dringende Renovierungen an dem Kliniksgebäude vorgenommen, das unter der sozialistischen Mangelwirtschaft deutlich gelitten hatte. Die Heizungsanlage wurde erneuert, die Patientenstationen wurden auf modernen Stand gebracht, die Photodermatologie wurde renoviert, und 1999-2000 wurde der Mitteltrakt mit Poliklinik, Hörsaal und Laborräumen grundsaniert. Gleichzeitig wurde ein moderner Eingriffsraum eingerichtet. Ausstehend ist allerdings noch die Schaffung von modernen Op-Räumen und die Renovierung der 1997 eingerichteten Tagesklinik. Ob diese Maßnahmen noch vorgenommen werden, ist derzeit ungewiss, denn die Dermatologie soll mit allen anderen Universitätskliniken bis Ende dieses Jahrzehnts in ein Neubauklinikum im Jenaer Vorort Lobeda umziehen.

Mit der Wende konnte auch die Ausstattung im klinischen Bereich und im Laborbereich erneuert werden. So konnten optimale Bedingungen etwa in der Phototherapie geschaffen werden, wo alle modernen lichttherapeutischen Methoden einschließlich der Hochdosis-UVA1-Therapie vorhanden sind. Zu
Tab. 2 Spezialsprechstunden der Dermatologischen Universitätsklinik Jena 2001

$\begin{array}{ll}\text { - Allergologische Sprechstunde } & \text { - Andrologische Sprechstunde } \\ \text { - Berufsdermatologische } & \text { - Psoriasis-Sprechstunde } \\ & \text { Sprechstunde } \\ \text { - Haarsprechstunde } & \text { - Kollagenosen-Sprechstunde } \\ \text { - Onkologische Sprechstunde } & \text { - Phlebologische Sprechstunde } \\ \text { - Photodermatologie } & \text { - Proktologische Sprechstunde } \\ \begin{array}{l}\text { (Lichtabteilung) } \\ \text { - Dermatologisch-rheumatologi- } \\ \text { sche Gemeinschaftssprech- }\end{array} & \text { - Sprechstunde für Genitaler- } \\ \text { stunde } & \text { krankungen der Frau } \\ \text { - Wundsprechstunde } & \text { - Extrakorporale Photopherese } \\ \text { - Neurodermitis-Sprechstunde } & \end{array}$

Tab. 3 Forschungsschwerpunkte der Dermatologischen Universitätsklinik Jena 2001

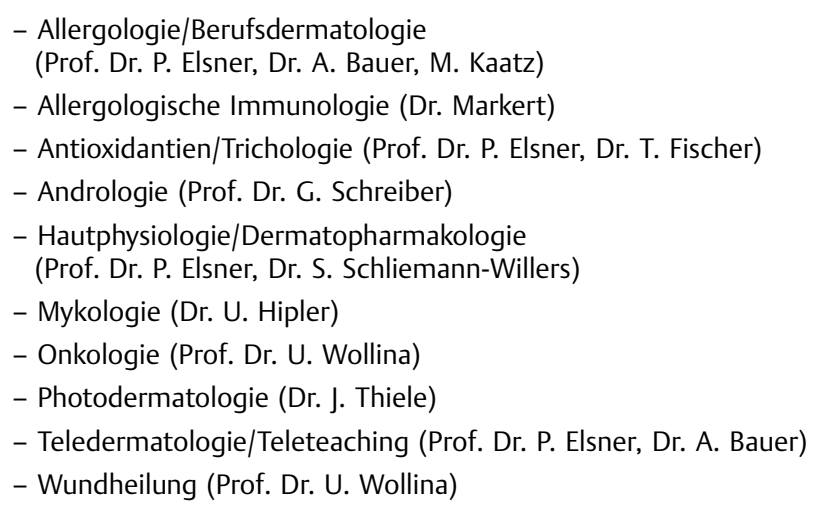

einem besonderen Schwerpunkt entwickelte sich die extrakorporale Photophorese. Als das Photophorese-Zentrum Thüringens hält die Klinik derzeit sogar zwei Geräte vor, die intensiv ausgelastet sind. Auch moderne Lasertherapie-Geräte konnten angeschafft werden. Leider hat sich in den letzten Jahren durch die Gesundheitspolitik der Bundesregierung und die Budgetreduktionen der Thüringer Landesregierung die wirtschaftliche Situation des Jenaer Universitätsklinikums drastisch verschlechtert, so dass auch für dringliche Nachhol-Investitionen derzeit keine Mittel vorhanden sind. Gleichzeitig kam es zu schmerzhaften Kürzungen im Personalbereich. Im Jahr 2001 verfügt die Jenaer Klinik für Dermatologie und dermatologische Allergologie über 14,5 Stellen für Ärzte und Naturwissenschaftler, 28,75 Stellen im Pflegebereich, 4,25 Stellen für medizinisch-technische Assistentinnen und 4,75 Stellen für Sekretariat und medizinische Dokumentation. Die Klinik hat - nach vorübergehendem Betten-Abbau - wieder 52 Betten, davon 38 im vollstationären und 14 im teilstationären Bereich.

Im ambulanten Bereich wurde im vergangenen Jahrzehnt der Schwerpunkt von der traditionellen allgemeinen Poliklinik auf Spezialsprechstunden verschoben, die den besonderen Anforderungen von Forschung und Lehre besser gerecht werden. Die im Jahr 2001 angebotenen Spezialsprechstunden finden sich in Tab. 2. Nach der Wende gewann die Jenaer dermatologische Klinik an klinischer Breite. Forschung, Lehre und Kran- 
kenversorgung in den zentralen dermatologischen Bereichen der Allergologie, der Dermatoonkologie und der Dermatochirurgie wurden gezielt ausgebaut. Daneben konnten Spezialbereiche wie die Trichologie und die interdisziplinäre Versorgung von Genitaldermatosen der Frau etabliert werden, die sich großen Zuspruchs der Patienten und der zuweisenden Ärzte erfreuen. In der Forschung wurden bestehende Schwerpunkte wie die Andrologie erhalten; es konnte aber auch eine Reihe von neuen Schwerpunkten etabliert werden (Tab. 3); Drittmitteileinwerbung und Publikationsaktivität zeigen eine erfreuliche Entwicklung [3].

\section{Perspektiven der Jenaer Dermatologie im 21. Jahrhundert}

Wenn die Entwicklung der Jenaer Dermatologie seit der Wende auch als durchweg positiv bezeichnet werden kann, so kann sie sich als Teil des Universitätsklinikums den zunehmend schwierigeren Rahmenbedingungen jedoch nicht entziehen. Zum gegenwärtigen Zeitpunkt ist noch nicht einzuschätzen, welche Auswirkungen die Einführung der „Diagnosis Related Groups“ (DRGs) auf die Dermatologie im stationären Bereich haben wird. Zu befürchten steht, dass die stationären Behandlungsmöglichkeiten für dermatologische Patienten zunehmend eingeschränkt werden und dass damit eine Verschlechterung der ärztlichen Versorgung, wie in den angelsächsischen Ländern vorexerziert, einhergeht. Offen ist auch, wie ein durch zahlreiche rechtliche Einschränkungen stranguliertes staatliches Universitätsklinikum erfolgreich mit einem zunehmend privatwirtschaftlich organisierten klinischen Umfeld konkurrieren kann. Und problematisch für die Entwicklung der Hochschulmedizin ist die massive Reduktion der Mittel für Lehre und Forschung durch die Landesregierung, da die Grundausstattung für die Drittmittelfähigkeit verloren geht.

Diese Probleme muss die Politik lösen. Als Anwälte unserer Patienten und als ihrer Verantwortung bewusste Staatsbürger dieses Staates müssen wir Dermatologen allerdings auf bedenkliche Entwicklungen aufmerksam machen. Es bleibt zu hoffen, dass einerseits die Qualität des deutschen Gesundheitssystems von der Politik als Standortvorteil im internationalen Wettbewerb erkannt und erhalten wird, und dass andererseits das hohe Innovationspotenzial der deutschen Hochschulmedizin auch im Interesse der wirtschaftlichen Entwicklung in Zukunft wieder adäquat gefördert wird. Die Jenaer Dermatologie ist jedenfalls bereit, ihre Chancen zum Nutzen der ihr anvertrauten Patienten aktiv wahrzunehmen.

\section{Literatur}

${ }^{1}$ Wollina U. 70 Jahre Universitäts-Hautklinik Jena. Zeitschr Dermatol 1994; 180: $58-62$

2 Wollina U, Bonitz R. 70 Jahre Universitäts-Hautklinik Jena - Tradition und Fortschritt. Hautarzt 1995; 46: 737 - 740

${ }^{3}$ Elsner P, Wollina U, Berndt U (Hrsg). Jahresbericht 2000. Jena: Dermatologische Klinik, 2001

\section{Prof. Dr. P. Elsner}

Klinik für Dermatologie und dermatologische Allergologie der Friedrich-Schiller-Universität Erfurter Straße 35

07740 Jena

E-mail: elsner@derma.uni-jena.de 